\title{
Smith-Lemli-Opitz syndrome: Clinical and biochemical findings in Brazilian patients. Scalco et al. Genetics and Molecular Biology (this issue)
}

\author{
John M. Opitz \\ Pediatrics (Medical Genetics), Human Genetics, Pathology, Obstetric \& Gynecology, University of Utah \\ School of Medicine, Salt Lake City, Utah, USA.
}

Scalco et al. present a clever diagnostic algorithm for nosologic application in the Smith-Lemli-Opitz" (SLO or RSH) syndrome based on their 11 biochemically confirmed patients and 34 others culled from prior publications. They are correct in inferring that none of the congenital anomalies they considered are specific to or pathognomonic of RSH syndrome, and that the greater the number of anomalies included in the calculation the greater the likelihood of effective nosologic discrimination and correct diagnosis.

While methodically sophisticated, this approach has two weaknesses. First, it is based on propositi biasing the sample to the most severe and of the phenotypic spectrum. While not common, mildly affected individuals with RSH syndrome are known. A preferable approach would be to base the discriminant analysis on secondarily ascertained cases including subsequently born sibs, indeed anybody in the family who is biochemically abnormal. Affected cousins, nieces and nephews in RSH families are known. In this way the phenotypic spectrum will be much more representative of all of the RSH individuals in the population. A beautiful example of such a study is that by Thomas et al. (1986) on the Fraser cryptophthalmos syndrome; the numbers of sibs of index cases is not large (maximum 23 in 124 families), but of exceptional value in projecting a less biased view of that disorder than one based on propositi.

Secondly, there is the issue of circularity due to inclusion in the analysis of all of the cases used for establishing the inventory of phenotypic manifestations. In a segregating disorder such as the RSH syndrome this problem could again be avoided by excluding index cases and basing the study on secondarily ascertained affected relatives.

Who are they? Anyone in the family of an index case with one or more than one RSH syndrome manifestation, diagnostically abnormal levels of cholesterol and 7DHC and/or the homozygous state of a DHCR7 mutation.

This view of the RSH syndrome will bring to light "cases" who would be excluded by the approach of Scalco et al., such as the biochemically confirmed fetus reported

Send correspondence to J.M. Opitz. Division of Medical Genetics, 2C412 SOM, 50 North Medical Drive, 84132 Salt Lake City, UT, USA. E-mail: john.opitz@ @sc.utah.edu. by Angle et al. (1988) with hydrops, uncharacteristic facial appearance and absence of $2 / 3$ to syndactyly who died at birth. Thus, fetal/pediatric geneticists have an extremely important role to play in any stillbirth and pediatric pathology program (q.v. Cernach et al., 2004) especially for the detection of segregating potentially lethal disorders presenting for the first time in a family. Fetal lethality in the RSH syndrome has been reported severally (Opitz et al., 2002; Putnam et al., 2005).

If Metherall is correct in his estimate of an almost 4\% gene frequency of mutant RSH alleles in the Utah CEPH families then the heterozygote and homozygote frequencies, respectively, are $\sim 8 \%$ and $1 / 625$ (BSJ Davies and JE Metherall, 2002, personal communication). Since we see only a small fraction of these homozygotes in fetal pathology and the pediatric genetics clinic they must die early during embryonic life. Since his allele frequency is identical in the normal and coronary heart disease population, it is evident that the carrier state of the RSH syndrome does not protect against coronary heart disease as the basis of a presumed heterozygote advantage.

What about the other 19 patients in the Scalco et al. study with normal levels of cholesterol and 7DHC? When all is said and done they clearly do not have the RSH syndrome, sensu stricto. In my opinion they are far more interesting than the 11 confirmed cases and deserve to be published in detail, or at least evaluated by an expert such as Richard Kelley or Malgorzata Nowaczyk before Scalco et al. begin the arduous task of testing their hypothesis that these biochemically normal individuals have a SHH protein mutation.

\section{References}

Cernach MC, Patricio FR, Galera MF, Moron AF and Brunoni D (2004) Evaluation of a protocol for postmortem examination of stillbirths and neonatal deaths with congenital anomalies. Pediatr Developm Pathol 7:335-341.

Opitz JM, Gilbert-Barness E, Ackerman J and Lowichik A (2002) Cholesterol and development: The RSH (Smith-LemliOpitz) syndrome and related conditions. Pediatr Pathol Mol Med 21:153-181. 
Putnam AR, Szakacs JG, Opitz JM and Byrne JLB (2005) Prenatal death in Smith-Lemli-Opitz/RSH syndrome. Am J Med Genet 138A:61-65.

Scalco FB, Otto PA, Brunetti IL, Cruzes VM and Moretti-Ferreira D (2006) Smith-Lemli-Opitz syndrome: Clinical and bio- chemical findings in Brazilian patients. Genet Mol Biol 29:429-436.

Thomas IT, Frías JL, Felix V, Sanchez de Leon L, Hernandez RA and Jones MC (1986) Isolated and syndromic cryptophthalmos. Am J Med Genet 25:85-98. 